\title{
Contemporary Management of Axillo-subclavian Arterial Injuries Using Data from the AAST PROOVIT Registry
}

\author{
Grahya Guntur ${ }^{1}$, Joseph J DuBose', Tiffany K Bee², Timothy Fabian², \\ Jonathan Morrison' ${ }^{1}$, David J Skarupa ${ }^{3}$, Kenji Inaba ${ }^{4}$, Rishi Kundi', \\ Thomas Scalea ${ }^{1}$, David V Feliciano ${ }^{1}$ and the AAST PROOVIT Study Group* \\ 'R Adams Cowley Shock Trauma Center, University of Maryland Medical System, Baltimore, MD, USA \\ 2University of Tennessee Health Sciences Center, Memphis, TN, USA \\ University of Florida Health, Jacksonville, FL, USA \\ ${ }^{4}$ Los Angeles County and University of Southern California Medical Center, Los Angeles, CA, USA
}

\begin{abstract}
Background: Endovascular repair has emerged as a viable repair option for axillo-subclavian arterial injuries in select patients; however, further study of contemporary outcomes is warranted.

Methods: The American Association for the Surgery of Trauma (AAST) PROspective Observational Vascular Injury Treatment (PROOVIT) registry was used to identify patients with axillo-subclavian arterial injuries from 2013 to 2019. Demographics and outcomes were compared between patients undergoing endovascular repair versus open repair.

Results: 167 patients were identified, with intervention required in 107 (64.1\%). Among these, 24 patients underwent open damage control surgery (primary amputation $=3$, ligation $=17$, temporary vascular shunt $=4$ ). The remaining 83 patients (91.6\% male; mean age $26.0 \pm 16)$ underwent either endovascular repair $(36,43.4 \%)$ or open repair $(47,56.6 \%)$. Patients managed with definitive endovascular or open repair had similar demographics and presentation, with the only exception being that endovascular repair was more commonly employed for traumatic pseudoaneurysms $(p=0.004)$. Endovascular repair was associated with lower 24 -hour transfusion requirements $(p=$ 0.012), but otherwise the two groups were similar with regards to in-hospital outcomes.

Conclusion: Endovascular repair is now employed in $>40 \%$ of axillo-subclavian arterial injuries undergoing repair at initial operation and is associated with lower 24-hour transfusion requirements, but otherwise outcomes are comparable to open repair.
\end{abstract}

Keywords: Axillo-subclavian Injury; Endovascular Repair; Vascular; Trauma

\section{Corresponding author:}

Joseph J DuBose, MD FACS FCCM FSVS, Professor of Surgery, R Adams Cowley Shock Trauma Center, University of Maryland Medical System, 22 South Greene Street, Room T4M14, Baltimore, MD 21201, USA.

Email: joseph.dubose@umm.edu

Presentation: This paper outlines work that was associated with data accepted for a poster presentation for the 2020 Annual American Association for the Surgery of Trauma meeting, that was held virtually in September 2020.

\begin{abstract}
*A full list of Study Group members and their affiliations appears at the end of the paper.

(C) 2021 CC BY 4.0 - in cooperation with Depts. of Cardiothoracic/ Vascular Surgery, General Surgery and Anesthesia, Örebro University Hospital and Örebro University, Sweden
\end{abstract}




\section{BACKGROUND}

Injuries to the axillary and subclavian arteries account for approximately $5-10 \%$ of civilian and military vascular injuries ${ }^{1,2}$. These injuries are, however, associated with challenging open exposures and reported morbidity and mortality rates ranging from $5 \%$ to $39 \%^{3,4}$. In this study, axillary and subclavian artery segments are grouped together since these two close segment neighbors are located in the junctional region of the forelimb that requires similar exposure considerations.

Traditional management of axillo-subclavian arterial injuries requiring intervention mandated open surgical exposure and repair. More recently, however, endovascular management has emerged as a viable alternative for treatment in select patients. When compared to open repair (OR), endovascular repair (ER) has been shown to be associated with decreased operative time, lower estimated blood loss, fewer iatrogenic injuries in the area of trauma, lower in-hospital mortality, and decreased rates of sepsis ${ }^{5-7}$. As endovascular technologies continue to evolve and their utilization continues to increase, there remains an important need to review subsequent outcomes. The purpose of this study was to compare in-hospital outcomes of ER and OR using a contemporary prospective vascular trauma registry.

\section{METHODS}

After Institutional Review Board Approval, the American Association for the Surgery of Trauma (AAST) PROspective Observational Vascular Injury Treatment (PROOVIT) registry was used to identify patients with axillo-subclavian arterial injuries from 2013 to 2019. This voluntary registry allows for collection of data from collaborating trauma centers and is open to all vascular injuries occurring at participating trauma centers. Patient demographics included age, gender, mechanism of injury, admission vital signs, Injury Severity Score (ISS), and Abbreviated Injury Scale score for each body region. Interventional data included operative procedures. Recorded outcomes included blood products transfused during hospital admission, complications of repair (thrombosis/stenosis, delayed amputation), hospital length of stay (LOS), and mortality. Patient demographics and in-hospital outcomes were compared between patients undergoing ER versus OR. Categorical variables were compared using Chi-square or Fisher exact tests, and continuous variables were compared using Student's $t$-test or Mann-Whitney rank-sum test. Significant results were designated with $p$-values less than 0.05 .

\section{Ethical Approval and Informed Consent}

Ethical approval to report these cases was given by the Institutional Review Board. Informed consent was not required.

\section{RESULTS}

At the time of the data pull for the present article, the PROOVIT registry contained 4357 patients. Among these, upper extremity injuries (subclavian, axillary, brachial, radial, and ulnar arteries) accounted for 1038 injuries, for a total of $23.8 \%$ (1038/4357). Among the upper extremity arterial injuries, axillo-subclavian accounted for $16.1 \%$ (167/1038) of upper extremity injuries (subclavian, axillary, brachial, radial, ulnar). From 2013 to 2019, there were 167 patients who sustained injuries to the axillary and subclavian arteries. Nonoperative management was used in 60 patients $(35.9 \%)$, and 24 patients were managed with initial open damage control. These groups of patients were not included in the comparison between patients who received either definitive ER or OR (Figures 1 and 2). In the 24 patients who received initial open damage control, there were eight who died $(33.3 \%)$ and three $(12.5 \%)$ who underwent amputations. Management in the remaining 21 patients included four $(16.7 \%)$ with vascular shunts, nine $(37.5 \%)$ with ligation, and eight $(33.3 \%)$ with initial ligation followed by delayed repair at a subsequent operation (Figure 2).

In the remaining 83 patients, definitive repair was by ER in $36(43.4 \%)$ and by OR in $47(56.6 \%)$ (Figure 1). Among the patients who received definitive treatment, there were $55(66.3 \%)$ with penetrating injuries. The types of injuries included $33(39.8 \%)$ transections, 21 $(25.3 \%)$ occlusions, $16(19.3 \%)$ pseudoaneurysms, and $19(22.9 \%)$ partial transections/flow-limiting defects (Table 1).

In patients treated with ER, the average age was 24.5 \pm 11 years, and $94.4 \%$ were males. At admission, $14.3 \%$ $(n=5)$ of the patients were hypotensive (systolic blood pressure $<90 \mathrm{~mm} \mathrm{Hg})$ and $68.8 \%(n=22)$ had an ISS $\geq 15$ (Table 1). In patients treated with OR, the average age was $27.5 \pm 16$ years, and $89.4 \%$ were males. At admission, $11.4 \%(n=5)$ of the patients were hypotensive (systolic blood pressure $<90 \mathrm{~mm} \mathrm{Hg}$ ) and $60.0 \%(n$ $=24$ ) had an ISS $\geq 15$ (Table 1).

When outcomes were compared between patients who underwent ER and OR, there was a significant difference with regard to total 24-hour transfusion requirements $(1.0 \pm 6$ units for ER vs $2.5 \pm 9$ units for $\mathrm{OR} ; p=0.012$ ). There were no deaths in the OR group and one in the ER group (mortality $=2.8 \%$ ). There was a need to intervene in $8.3 \%$ of patients $(n=3)$ undergoing ER and $8.5 \%$ of patients $(n=4)$ undergoing OR $(p=1.00)$. There were two $(5.6 \%)$ delayed amputations in the ER group and one $(2.1 \%)$ in the OR group $(p=0.576)($ Table 2$)$.

\section{DISCUSSION}

With a total of 167 patients, this study is one of the largest in the trauma field comparing the use of ER and 


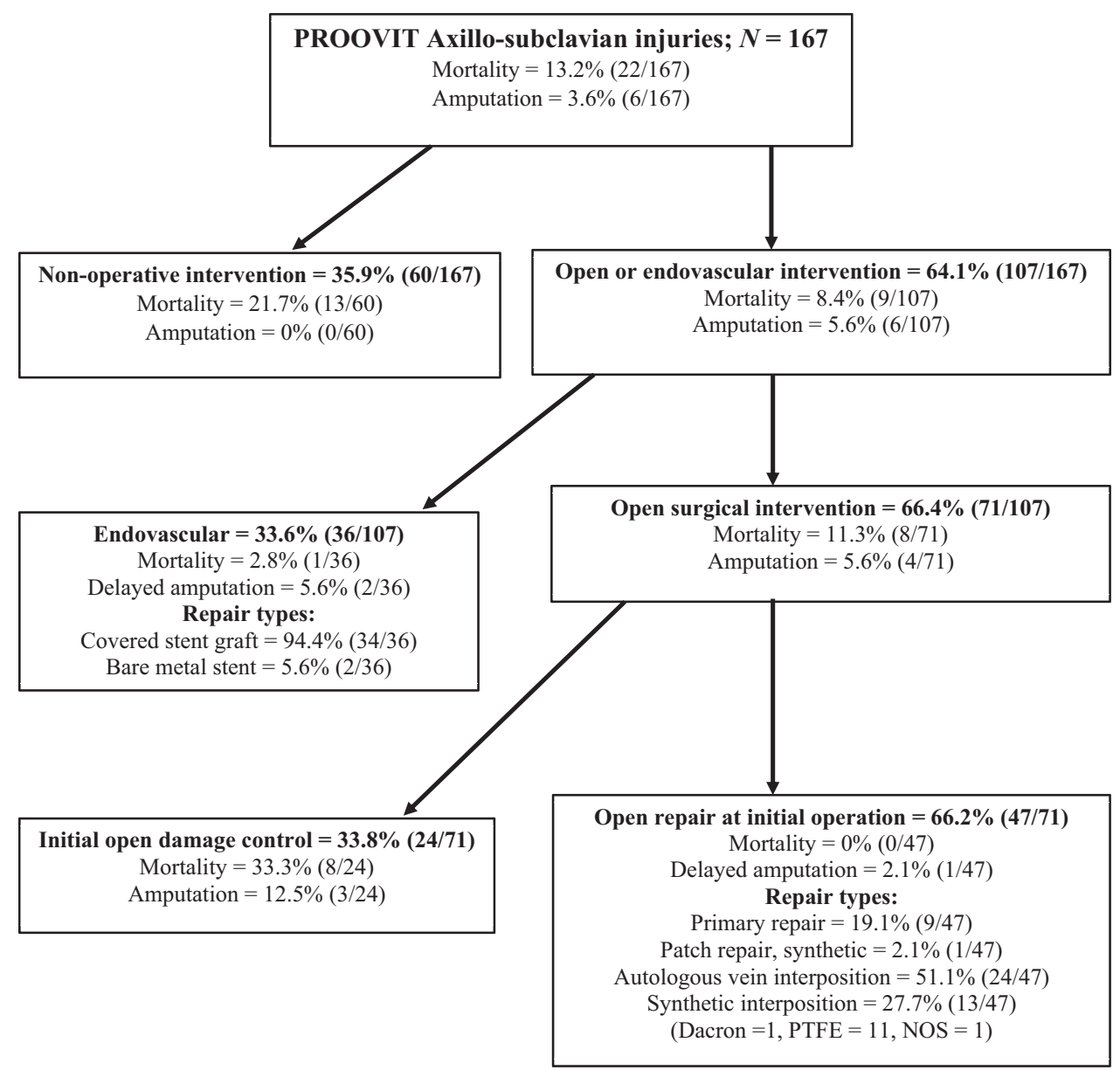

Figure 1 Breakdown of all patients used from PROOVIT data. $N=167$ represents total number of patients used in this study. Mortality rate and amputation rate are represented in each group of patients. NOS, not otherwise specified; PTFE, polytetrafluoroethylene.

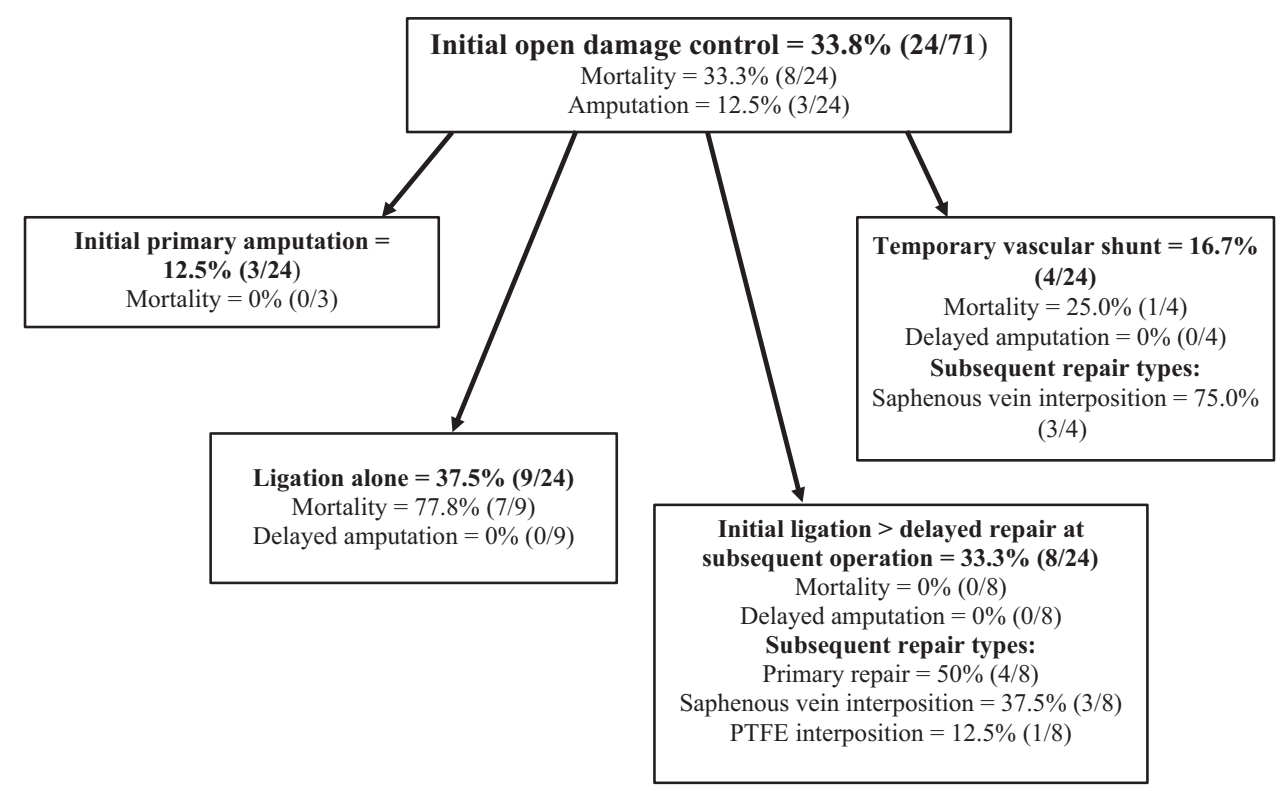

Figure 2 Breakdown of patients who underwent initial open damage control. Mortality rate and amputation rate are represented in each group of patients. PTFE, polytetrafluoroethylene. 
Table 1 Demographic and clinical data of patient groups.

\begin{tabular}{|c|c|c|c|c|}
\hline & $\begin{array}{c}\text { Total } \\
(N=83)\end{array}$ & $\begin{array}{l}\text { Open Repair } \\
(N=47)\end{array}$ & $\begin{array}{l}\text { Endovascular Repair } \\
\qquad(N=36)\end{array}$ & $p$-value \\
\hline Age, years (median $\pm 1 Q R$ ) & $26.0 \pm 16$ & $27.5 \pm 16$ & $24.5 \pm 11$ & 0.979 \\
\hline Male, $\%(n / N)$ & $91.6 \%(76 / 83)$ & $89.4 \%(42 / 47)$ & $94.4 \%(34 / 36)$ & 0.341 \\
\hline Penetrating, \% ( $n / N)$ & $66.3 \%(55 / 83)$ & $66.0 \%(31 / 47)$ & $66.7 \%(24 / 36)$ & 0.976 \\
\hline Transection, \% (n/N) & $39.8 \%(33 / 83)$ & $44.7 \%(21 / 47)$ & $33.3 \%(12 / 36)$ & 0.295 \\
\hline Occlusion, \% (n/N) & $25.3 \%(21 / 83)$ & $21.3 \%(10 / 47)$ & $30.6 \%(11 / 36)$ & 0.335 \\
\hline Partial transection/flow-limiting defect, \% ( $n / N)$ & $22.9 \%(19 / 83)$ & $27.7 \%(13 / 47)$ & $16.7 \%(6 / 36)$ & 0.237 \\
\hline Pseudoaneurysm, \% (n/N) & $19.3 \%(16 / 83)$ & $8.5 \%(4 / 47)$ & $33.3 \%(12 / 36)$ & 0.004 \\
\hline SBP on admission (median \pm IQR) & $123 \pm 51$ & $128 \pm 49$ & $119 \pm 53$ & 0.596 \\
\hline Hypotension on admission, \% ( $n / N)$ & $12.7 \%(10 / 79)$ & $11.4 \%(5 / 44)$ & $14.3 \%(5 / 35)$ & 0.743 \\
\hline ISS (median \pm IQR) & $18 \pm 14$ & $17 \pm 17$ & $20 \pm 15$ & 0.163 \\
\hline ISS $\geq 15, \%(n / N)$ & $63.9 \%(46 / 72)$ & $60.0 \%(24 / 40)$ & $68.8 \%(22 / 32)$ & 0.442 \\
\hline Head AIS $\geq 3, \%(n / N)$ & $20.6 \%(13 / 63)$ & $22.9 \%(8 / 35)$ & $17.9 \%(5 / 28)$ & 0.626 \\
\hline Chest AIS $\geq 3, \%(n / N)$ & $71.4 \%(50 / 70)$ & $63.2 \%(24 / 38)$ & $81.3 \%(26 / 32)$ & 0.095 \\
\hline Abdomen AIS $\geq 3, \%(n / N)$ & $9.4 \%(6 / 64)$ & $5.6 \%(2 / 36)$ & $14.3 \%(4 / 28)$ & 0.391 \\
\hline Extremity AIS $\geq 3, \%(n / N)$ & $41.2 \%(28 / 68)$ & $50.0 \%(19 / 38)$ & $30.0 \%(9 / 30)$ & 0.096 \\
\hline
\end{tabular}

Data is represented as either median $\pm I Q R$ or percentage $(n / N)$. $p$-value $<0.05$ is considered significant. AIS, Abbreviated Injury Score; IQR, interquartile range; $n$, number of patients with each clinical characteristic; $N$, number of patients in total, in the OR group or in the ER group.

Table 2 In-hospital outcomes of patients in ER and OR groups.

\begin{tabular}{lcccc}
\hline & $\begin{array}{c}\text { Total } \\
(N=83)\end{array}$ & $\begin{array}{c}\text { Open Repair } \\
(N=47)\end{array}$ & $\begin{array}{c}\text { Endovascular Repair } \\
(N=36)\end{array}$ & $p$-value \\
\hline Total PRBCs first 24 hours, units (median \pm IQR) & $2.0 \pm 7$ & $2.5 \pm 9$ & $1.0 \pm 6$ & 0.012 \\
Need to re-intervene on initial repair, \% $(n / N)$ & $8.4 \%(7 / 83)$ & $8.5 \%(4 / 47)$ & $8.3 \%(3 / 36)$ & 1.000 \\
Thrombosis of repair, \% $(n / N)$ & $4.8 \%(4 / 83)$ & $4.3 \%(2 / 47)$ & $5.6 \%(2 / 36)$ & 1.000 \\
Flow-limiting stenosis, \% $(n / N)$ & $1.2 \%(1 / 83)$ & $0 \%(0 / 47)$ & $2.8 \%(1 / 36)$ & 0.434 \\
Infection resulting in need to re-operate, \% $(n / N)$ & $0 \%(0 / 83)$ & $0 \%(0 / 47)$ & $0 \%(0 / 36)$ & $N / A$ \\
Delayed amputation, \% $(n / N)$ & $3.6 \%(3 / 83)$ & $2.1 \%(1 / 47)$ & $5.6 \%(2 / 36)$ & 0.576 \\
Stroke related to vascular injury or repair, \% $(n / N)$ & $2.4 \%(2 / 83)$ & $2.1 \%(1 / 47)$ & $2.8 \%(1 / 36)$ & 1.000 \\
Hospital LOS (median \pm IQR) & $8.0 \pm 13$ & $7.5 \pm 13$ & $9.0 \pm 15$ & 0.864 \\
ICU LOS (median \pm IQR) & $3.0 \pm 5$ & $3.0 \pm 5$ & $2.0 \pm 4$ & 0.629 \\
Mortality, \% $(n / N)$ & $1.2 \%(1 / 83)$ & $0 \%(0 / 47)$ & $2.8 \%(1 / 36)$ & 0.434 \\
\hline
\end{tabular}

Data is represented as either median $\pm 1 Q R$ or percentage $(n / N)$. $p$-value $<0.05$ is considered significant. IQR, interquartile range; $n$, number of patients with each clinical characteristic; N, number of patients in total, in the OR group or in the ER group. PRBC, packed red blood cells; LOS, length of stay; ICU, Intensive care Unit.

OR for axillo-subclavian arterial injuries. The PROOVIT registry includes multicenter data specific to vascular injury which captures variables and outcomes not available in previous retrospective studies utilizing dual center or NTDB data. By using data from the PROOVIT registry over a seven-year period, this study assessed the outcomes of axillo-subclavian injuries after ER or OR.

The historic approach to axillo-subclavian injuries has been OR, and successful treatment relies on recognition of the severity of the injury and rapid control of hemorrhage or restoration of flow ${ }^{8,9}$. To date, ER has been reserved for patients who are more hemodynamically stable, but it is becoming more widely used in other patients as more advantages are discovered. These have been documented in multiple reviews of injuries to the carotid artery, abdominal aorta, iliac artery, femoral artery, and the axillo-subclavian arteries ${ }^{10-14}$.

Furthermore, there have been studies showing improved outcomes with ER for axillo-subclavian arterial injuries. In 2011, Shalhub et al. ${ }^{15}$ performed a retrospective review of 34 patients with blunt thoracic outlet arterial injuries in which 12 were managed with endovascular repair. They demonstrated that patients treated with ER had shorter operative times, less blood loss, and less morbidity. Similarly, a retrospective study by Branco et al. ${ }^{16}$ in 2016 reviewed 153 patients with axillo-subclavian injuries over an 11-year period. There were 72 patients matched based on demographics and clinical data, with 18 patients managed with ER and 54 with OR. Patients who underwent ER had significantly lower in-hospital mortality and lower rates of surgical 
site infections and sepsis. Another study by Matsagkas et al. ${ }^{17}$ investigated the use of ER in seven patients with blunt trauma to the axillary and subclavian arteries. They found that endovascular technique for blunt injuries was reliable with no procedure-related complications during the median hospital stay of 22 days and there was a $0 \%$ mortality rate. Finally, a retrospective review by Waller et al. ${ }^{18}$ found that, while axillary and subclavian artery injuries still require open exposures and repairs, endovascular repairs are more effective for pseudoaneurysms.

The current review documents a continuing increase in the use of ER for axillo-subclavian injuries. In the group of 83 patients who received definitive treatment, $36(43.4 \%)$ were managed with ER. This is similar to other studies over the past decade in which $60 \%$ and $42.9 \%$ of patients with axillo-subclavian injuries were managed with $\mathrm{ER}^{15,19}$.

Overall mortality in this review was $13.2 \%$. Patients who were hemodynamically unstable underwent initial open damage control and had a mortality rate of $33.3 \%$. In the remaining patients who received definitive treatment with either ER or OR, the mortality rate was only $1.2 \%$ as previously noted. In addition, there was a low number of amputations in both treatment groups. The only significant outcome of this review was that there were less blood transfusions in the ER group; however, it is clear that ER is comparable to OR for axillo-subclavian artery injuries when considering mortality, thrombosis of repair, flow-limiting stenosis, infection rates, amputation rate, stroke, and LOS.

As with every study, there were a number of limitations. First, this was a retrospective review of data from the PROOVIT registry. Databases such as the PROOVIT registry have some limits in information provided for individual patients and in the variability in contribution from the patients enrolled in this unfunded and voluntary effort. Furthermore, patients had definitive treatment with either ER or OR based on their hemodynamic stability and feasibility of intervention in this nonrandomized review. In addition, these patients were not matched based on demographic or clinical variables such as age, type of injury, severity of injury, and blood pressure on admission for statistical analysis. Also, data on whether patients needed a thoracotomy, sternotomy, or laparotomy and on procedural times, longer term outcomes outside of the hospitalization, out of hospital outcomes, estimated blood loss from all injuries, precise amount blood loss from the axillo-subclavian injury, and Acute Respiratory Distress Syndrome (ARDS) were not available. This restricts the ability of the results in this review to be compared to those in previous studies $^{1,15,16}$. Finally, indications for initial damage control and exact outcome metrics were not available, which prevented this subset of patients from being compared to the endovascular treatment group.

\section{CONCLUSION}

The current review documents a continuing increase in the use of ER for axillo-subclavian arterial injuries. In the group of 83 patients who received definitive treatment, $36(43.4 \%)$ were managed with ER. Overall mortality in this review was $13.2 \%$. Patients who were hemodynamically unstable underwent initial open damage control and had a mortality rate of $33.3 \%$. In the remaining patients who received definitive treatment with either ER or OR, the mortality rate was only $1.2 \%$ as previously noted. The only significant outcome of this review was that there were less blood transfusions in the ER group; however, it is clear that ER is comparable to OR for axillo-subclavian arterial injuries when considering mortality, thrombosis of repair, flow-limiting stenosis, infection rates, amputation rate, stroke, and LOS. Additional studies will be required to assess specific management techniques depending on location of vascular injury and accessibility of injury.

\section{Ethics Statement}

(1) All the authors mentioned in the manuscript have agreed to authorship, read and approved the manuscript, and given consent for submission and subsequent publication of the manuscript.

(2) The authors declare that they have read and abided by the JEVTM statement of ethical standards including rules of informed consent and ethical committee approval as stated in the article.

\section{Conflicts of Interest}

The authors declare that they have no conflicts of interest.

\section{Funding}

The authors received no financial support for the research, authorship, and/or publication of this article.

\section{The AAST PROOVIT Study Group}

John Sharpe, Tiffany Bee, Timothy Fabian: University of Tennessee Health Sciences Center, Memphis, TN, USA; Jonny Morrison, David Feliciano, Thomas M Scalea: University of Maryland, R Adams Cowley Shock Trauma Center, Baltimore, MD, USA; David Skarupa, Jennifer A Mull, Yohan Diaz Zuniga: University of Florida - Jacksonville, Jacksonville, FL, USA; Jeanette M Podbielski, Garrett Jost: University of Texas Health Sciences Center - Houston, Houston, TX, USA; Richard D Catalano, Ahmed M Abou-Zamzam Jr, Xian LuoOwen: Loma Linda University Medical Center, Loma Linda, CA, USA; Jennie Kim, Kenji Inaba: Los Angeles County and University of Southern California Hospital, Los Angeles, CA, USA; Nathaniel Poulin: East Carolina Medical Center, Benson, North Carolina, USA; John 
Myers, Michael Johnson, Kristin Rocchi: The University of Texas Health Sciences Center at San Antonio, San Antonio, TX, USA; John K Bini, Joshua Pringle, Karen Herzing, Kailey Nolan: Wright State Research Institute - Miami Valley Hospital, Dayton, OH, USA; Ramyar Gilani, Tikesha Smith, Reginva Knight: Ben Taub General Hospital/Baylor College of Medicine, Houston, TX, USA and JBSA Fort Sam Houston, TX, USA; Peter Hammer: Indiana University School of Medicine, Indianapolis, IN, USA; Scott T Trexler: San Antonio Military Medical Center / US Army Institute of Surgical Research, San Antonio, TX, USA; Nicholas Namias: Ryder Trauma Center, University of Miami/Jackson Memorial, Miami, FL, USA; Juan Asensio: Creighton University School of Medicine, Omaha, Nebraska, USA; Joseph M Galante, Misty Humphries: University of California - Davis, Sacramento, CA, USA; Ravi R Rajani: Emory University School of Medicine - Grady Memorial Hospital, Atlanta, GA, USA; George Dulabon, Riyad Karmy-Jones: Peace Health Southwest Washington Medical Center, Vancouver, Washington, USA; Andreas Larentzakis, George Velmahos: Massachusetts General Hospital, Boston, Massachusetts, USA; Suresh Agarwal: University of Wisconsin, Madison, WI, USA; Jayraan Badiee, Michael Sise: Scripps Mercy Hospital, San Diego, CA, USA; Alan Cook, Annette Taylor, John Zumbuhi, Antonia Greigo: Chandler Regional Medical Center, Chandler, AZ, USA; Fausto Y Vinces, Salvatore Docimo: Lutheran Medical Center, Brooklyn, New York, USA; Matthew M Carrick, Kathy Rodkey: Medical City Plano, Plano, TX, USA; Sameer Hirji, Reza Askari: Brigham and Women's Hospital, Boston, MA, USA; Forrest O Moore, Richard Butler: John Peter Smith Hospital, Fort Worth, TX, USA.

\section{REFERENCES}

[1] Xenos ES, Freeman M, Stevens S, Cassada D, Pacanowski J, Goldman M. Covered stents for injuries of subclavian and axillary arteries. J Vasc Surg. 2003;38(3):451-4.

[2] Fox CJ, Gillespie DL, O'Donnell SD, et al. Contemporary management of wartime vascular trauma. J Vasc Surg. 2005;41(4):638-44.

[3] Danetz JS, Cassano AD, Stoner MC, Ivatury RR, Levy MM. Feasibility of endovascular repair in penetrating axillosubclavian injuries: a retrospective review. J Vasc Surg. 2005;41(2):246-54.

[4] Demetriades D, Chahwan S, Gomez H, et al. Penetrating injuries to the subclavian and axillary vessels. J Am Coll Surg. 1999;188(3):290-95.
[5] Reuben BC, Whitten MG, Sarfati M, Kraiss LW. Increasing use of endovascular therapy in acute arterial injuries: analysis of the National Trauma Data Bank. J Vasc Surg. 2007;46(6):1222-6.

[6] du Toit DF, Coolen D, Lambrechts A, de V Odendaal J, Warren BL. The endovascular management of penetrating carotid artery injuries: long-term follow-up. Eur J Vasc Endovasc Surg. 2009;38(3):267-72.

[7] Branco BC, DuBose JJ, Zhan LX, et al. Trends and outcomes of endovascular therapy in the management of civilian vascular injuries. J Vasc Surg. 2014;60(5):1297-307.

[8] Graham JM, Mattox KL, Feliciano DV, DeBakey ME. Vascular injuries of the axilla. Ann Surg. 1982;195(2): 232-8.

[9] Graham JM, Feliciano DV, Mattox KL, Beall AC Jr, DeBakey ME. Management of subclavian vascular injuries. J Trauma. 1980;20(7):537-44.

[10] Velmahos GC, Chahwan S, Hanks SE, et al. Angiographic embolization of bilateral internal iliac arteries to control life-threatening hemorrhage after blunt trauma to the pelvis. Am Surg. 2000;66(9):858-62.

[11] Johnson CA. Endovascular management of peripheral vascular trauma. Semin Intervent Radiol. 2010;27(1):38-43.

[12] DuBose J, Recinos G, Teixeira PG, Inaba K, Demetriades D. Endovascular stenting for the treatment of traumatic internal carotid injuries: expanding experience. J Trauma. 2008;65(6):1561-6.

[13] Shalhub S, Starnes BW, Tran NT, et al. Blunt abdominal aortic injury. J Vasc Surg. 2012;55(5):1277-85.

[14] Danetz JS, Cassano AD, Stoner MC, Ivatury RR, Levy MM. Feasibility of endovascular repair in penetrating axillosubclavian injuries: a retrospective review. J Vasc Surg. 2005;41(2):246-54.

[15] Shalhub S, Starnes BW, Hatsukami TS, Karmy-Jones R, Tran NT. Repair of blunt thoracic outlet arterial injuries: an evolution from open to endovascular approach. J Trauma. 2011;71(5):E114-21.

[16] Branco BC, Boutrous ML, DuBose JJ, et al. Outcome comparison between open and endovascular management of axillosubclavian arterial injuries. J Vasc Surg. 2016;63(3):702-709.

[17] Matsagkas M, Kouvelos G, Peroulis M, Xanthopoulos D, Bouris V, Arnaoutoglou E. Endovascular repair of blunt axillo-subclavian arterial injuries as the first line treatment. Injury. 2016;47(5):1051-6.

[18] Waller CJ, Cogbill TH, Kallies KJ, et al. Contemporary management of subclavian and axillary artery injuries A Western Trauma Association multicenter review. J Trauma Acute Care Surg. 2017;83(6):1023-31.

[19] Carrick MM, Morrison CA, Pham HQ, et al. Modern management of traumatic subclavian artery injuries: a single institution's experience in the evolution of endovascular repair. Am J Surg. 2010;199(1):28-34. 